\title{
Body centered photonic crystal
}

\author{
K.B.S.K.B. Jayawardana, K.A.I.L. Wijewardena Gamalath ${ }^{*}$ \\ Department of Physics, University of Colombo, Colombo3, Sri Lanka \\ imalie@phys.cmb.ac.Ik
}

Keywords: Photonic crystals, body centered cubic, plane wave expansion method, mode field distributions, band gap, filling factor, gap width to midgap frequency ratio.

\begin{abstract}
The photonic energy bands of body centered cubic photonic crystals formed from $\mathrm{SiO}_{2}, \mathrm{GaP}, \mathrm{Si}, \mathrm{InAs}, \mathrm{GaAs}, \mathrm{InP}, \mathrm{Ge}$ and $\mathrm{BaSrTiO}_{3}$ dielectric spheres drilled in air and air holes drilled in these dielectric mediums were calculated using the plane wave expansion method. The filling factor for each dielectric material was changed until a complete energy gap was obtained and then the density of states was calculated. There were no complete band gaps for air spheres drilled in these eight dielectric mediums. The lattice constants were determined by using wavelengths in the region $1550 \mathrm{~nm}-1 \mathrm{~nm}$. The variation of the band gap widths with the filling factor and the variation of gap width to midgap frequency ratios with dielectric contrast were investigated. The largest band gap width of 0.021 for normalized frequency was obtained for $\mathrm{GaP}$ for the filling factor of 0.0736 . The mode filed distributions were obtained by guiding a telecommunication wave with $1.55 \mu \mathrm{m}$ wavelength through a $3 \times 3 \times 3$ photonic cell formed from $\mathrm{GaP}$ spheres in air with a filling factor of 0.0736 for transverse electric and magnetic modes.
\end{abstract}

\section{INTRODUCTION}

From the commencement of the telecommunication industry, engineers and scientists have been compelled to control electromagnetic waves and a new type of periodic dielectric structures formed artificially known as photonic crystals gave the solution to most of their problems. These crystals also revolutionized the physics of optics and signal transmissions. Photonic crystals are the optical analogue of semiconductors, possessing a photonic band gap (PBG), as opposed to an electronic band gap, where the propagation of photons is prohibited for energies that lie within the gap. When electromagnetic waves interact with these crystals, they periodically modulate the dielectric constant. The photonic band gap may exist only in the direction over which the dielectric constant is modulated. It is possible to assign scattering centers for composite materials, where light propagate gradually. In photonic crystals, these scattering centers are periodically arranged, so that the light scatters coherently. In this situation due to interference some frequencies will not be able to propagate giving rise to disallowed or sanctioned bands. Under certain conditions, there will be regions of forbidden frequencies for all the directions of propagation, and in such cases photonic crystals have full photonic band gaps. Only the three dimensional photonic crystals may possess a complete photonic band gap in all directions, which is significant for optical or optoelectronic device operations. These band gaps would give rise to optical phenomena such as inhibition of spontaneous emissions, high-reflecting omni-directional mirrors and low-loss-wave guiding. The scattering strength in the crystal may be assessed qualitatively by the refractive index contrast between the two materials, specifically by the ratio of their refractive indices.

The three dimensional photonic band gap structures came into field with a theoretical study by Bykov with his speculations on two or three-dimensional optical structures [1]. In 1979 Ohtaka came up with a concept of three-dimensional photonic crystal composed with identical dielectric spheres ordered in a three-dimensional lattice and discussed a method to calculate the band structure [2]. Yablonovitch [3] and John [4] published two milestone papers on photonic crystals in 1987. Both these papers were related to high dimensional photonic crystals. In 1991, Yablonovitch demonstrated the first three-dimensional photonic band-gap in the microwave regime [5]. In 2008, Joannopoulos and his research team engendered a software package to run in Linux operating 
system using finite difference time domain (FDTD) method to simulate photonic band gaps in all $1 \mathrm{D}, 2 \mathrm{D}$ and $3 \mathrm{D}$ situations [6].

The plane wave expansion method applicable to any type of non-dispersive dielectric functions was used as the numerical technique in the present work in modeling and simulating the band structures of three dimensional photonic crystals with body centered cubic (BCC) lattice formed from $\mathrm{SiO}_{2}$, GaP, $\mathrm{Si}$, InAs, GaAs, InP, Ge and $\mathrm{BaSrTiO}_{3}$ spheres drilled in air and air holes drilled in these dielectric mediums. For the periodic dielectric function, the magnetic field vector was expanded using Bloch theorem leading to eigenvalue equation in matrix form. Standard eigenvalue equation was solved for the lattice geometries and the energy bands of the eight materials were obtained. The filling factor for each dielectric material was changed until a complete energy gap was obtained. There were no complete band gaps for air spheres drilled in these eight dielectric mediums. Density of states (DOS) was calculated for the photonic lattice with a complete band gap. The effects of the parameters on the band gaps were studied. Optimum band gap materials, filling factors and the lattice constants for BCC structures that can be used in radio frequency, extremely high frequency, super high frequency, ultra high frequency and very high frequency are given. The mode field distributions for TE and TM mode were obtained when an electromagnetic wave of telecommunication wavelength passes through $3 \times 3 \times 3$ BCC lattice formed from GaP spheres of radius $0.25 a$ drilled in air.

\section{PLANE WAVE EXPANSION METHOD}

The propagation of light in a photonic crystal is governed by the four Maxwell equations. By combining the source-free Faraday's and Ampere's laws at a fixed angular frequency $\omega$ that is with harmonic time dependence of the electric field $\boldsymbol{E}(\boldsymbol{r}, t)=\boldsymbol{E}(\boldsymbol{r}) e^{-i \omega t}$ and magnetic field strength $\boldsymbol{H}(\boldsymbol{r}, t)=\boldsymbol{H}(\boldsymbol{r}) e^{-i \omega t}$ leads to the master equation for a periodic dielectric structure:

$$
\nabla \times \frac{1}{\varepsilon(r)} \nabla \times \boldsymbol{H}=\frac{\omega^{2}}{c^{2}} \boldsymbol{H}(r)
$$

Here the photonic crystal was considered to be a macroscopic, homogeneous, isotropic dielectric material without placed charges, current densities and dispersion. The dielectric constant $\varepsilon(\boldsymbol{r})$ is considered as real and for isotropic dielectric materials $\mu(r) \approx 1$.

There are several methods for solving a photonic band structure such as plane wave expansion method, finite element method, finite difference time domain method and Bloch wave MOM method. The most customary and popular methods are the plane wave expansion method (PWE) and finite difference time domain method (FDTD). According to the Bloch theorem, a plane wave in a periodic structure will be modulated by the periodicity in the medium. Hence, the magnetic field $\boldsymbol{H}$ can be expressed by a periodic envelope function multiplied by a plane wave:

$$
\boldsymbol{H}(\boldsymbol{r})=h(\boldsymbol{r}) e^{i \boldsymbol{k} \cdot \boldsymbol{r}} \hat{e}_{\boldsymbol{k}}, \quad h(\boldsymbol{r})=h\left(\boldsymbol{r}+\boldsymbol{R}_{l}\right)
$$

$h(\boldsymbol{r})$ is the periodic envelop function with the periodicity of the arbitrary lattice vector $\boldsymbol{R}_{l}$ and $\hat{e}_{\boldsymbol{k}}$ is a unit vector perpendicular to the wave vector $\boldsymbol{k}$ and parallel to the magnetic vector $\boldsymbol{H}$. In plane wave expansion method, a set of plane waves are used to expand the periodic functions in a Fourier series. Because of the periodic nature of photonic crystals, the magnetic field is expanded into a sum of plane waves in the reciprocal space giving an arbitrary spatial frequency call reciprocal lattice vector $\boldsymbol{G}_{i}=h_{i} \boldsymbol{b}_{i}$ in terms of basis vectors $\boldsymbol{b}_{i}$ and a set of integers $h_{i}$ :

$$
\boldsymbol{H}(\mathrm{r})=e^{i \boldsymbol{k} \cdot \boldsymbol{r}} \hat{e}_{\boldsymbol{k}} \sum_{\boldsymbol{G}_{i}} h(\boldsymbol{r}) e^{i \boldsymbol{G} \cdot \boldsymbol{r}}=\sum_{\boldsymbol{G}_{i}, \lambda} h\left(\boldsymbol{G}_{i}, \lambda\right) e^{i(\boldsymbol{k}+\boldsymbol{G}) \cdot \boldsymbol{r}} \hat{e}_{\lambda, \boldsymbol{k}+\boldsymbol{G}_{i}}
$$

where $\boldsymbol{G}_{i}$ is the frequency component, $\lambda$ represents the two axes in the perpendicular plane and $\hat{e}_{\lambda}$ is the unit vector along these axes. $h\left(\boldsymbol{G}_{i}, \lambda\right)$ is the amplitude component of the envelop function 
along the unit vector. $\hat{e}_{\lambda, \boldsymbol{k}+\boldsymbol{G}_{i}}$ are two orthogonal unit vectors perpendicular to $\boldsymbol{k}+\boldsymbol{G}_{i}$. The dielectric function of the structure is similarly expanded in terms of reciprocal lattice vectors:

$$
\varepsilon(\boldsymbol{r})=\sum_{G_{i}} \varepsilon\left(\boldsymbol{G}_{i}\right) e^{i \boldsymbol{G} \cdot \boldsymbol{r}}, \quad \varepsilon(\boldsymbol{G})=\frac{1}{A} \iint \varepsilon(\boldsymbol{r}) e^{-i \boldsymbol{G} \cdot \boldsymbol{r}} d^{2} r
$$

Here the integration is performed over the area $A$ of one lattice unit cell. Substituting equations 3 and 4 in 1 :

$$
\sum_{G^{\prime}}|\vec{k}+\vec{G}|\left|\vec{k}+\overrightarrow{G^{\prime}}\right| \varepsilon^{-1}\left(\vec{G}-\overrightarrow{G^{\prime}}\right)\left[\begin{array}{cc}
\hat{e}_{2} \cdot \hat{e}_{2}^{\prime} & -\hat{e}_{2} \cdot \hat{e}_{1}^{\prime} \\
-\hat{e}_{1} . \hat{e}_{2}^{\prime} & \hat{e}_{1} \cdot \hat{e}_{1}^{\prime}
\end{array}\right]\left[\begin{array}{l}
h_{1}^{\prime} \\
h_{2}^{\prime}
\end{array}\right]=\left[\begin{array}{cc}
M_{1} & M_{2} \\
M_{3} & M_{4}
\end{array}\right]\left[\begin{array}{l}
h_{1}\left(G^{\prime}\right) \\
h_{2}\left(G^{\prime}\right)
\end{array}\right]=\frac{\omega^{2}}{c^{2}}\left[\begin{array}{l}
h_{1} \\
h_{2}
\end{array}\right]
$$

Unlike in one dimensional and two dimensional simplifications, master eigenvalue equation does not exist in three dimensional case. Hence the two unit vectors $\hat{e}_{1, k+G}$ and $\hat{e}_{2, k+G}$ which are perpendicular to $\boldsymbol{k}+\boldsymbol{G}$ vector had to be calculated. Periodic dielectric function in real space can be simplified as,

$$
\varepsilon(r)= \begin{cases}\varepsilon_{a} & r<R \\ \varepsilon_{b} & r>R\end{cases}
$$

where $\varepsilon_{a}$ and $\varepsilon_{b}$ refer to the dielectric constants of the localized medium and background respectively. Fourier transformation of the dielectric function can be further simplified to,

$$
\varepsilon(\boldsymbol{G})= \begin{cases}f \varepsilon_{a}+(1-f) \varepsilon_{b} & \text { for } \boldsymbol{G}=0 \\ \left(\varepsilon_{a}-\varepsilon_{b}\right) S(\boldsymbol{G}) & \text { for } \boldsymbol{G} \neq 0\end{cases}
$$

where $f$ is the filling factor, defined as the fraction of area occupied by the localized medium in one unit cell. The structure factor $S(\boldsymbol{G})$ relying on the geometry of the localized medium and the lattice structures is given by,

$$
S(\boldsymbol{G})=\frac{1}{A} \int_{A_{d}} e^{-i \boldsymbol{G} \cdot \boldsymbol{r}} d^{2} r .
$$

The structure factor for a three dimensional sphere with radius $R$ is given by

$$
S(G)=3 f\left[\frac{\sin (G R)-G R \cos (G R)}{(G R)^{3}}\right] .
$$

Any two different modes of magnetic and electric vectors are orthogonal to each other i.e. $<H_{i} \mid H_{j}>=\delta_{i j}$ and degenerate modes are not necessarily orthogonal. The electric $\left(\mathrm{E}_{E D}\right)$ and magnetic $\left(\mathrm{E}_{M D}\right)$ mode energies are:

$$
\begin{aligned}
& \mathrm{E}_{E D}=\frac{1}{8 \pi} \int \frac{1}{\varepsilon(r)}|D(r)|^{2} d r \\
& \mathrm{E}_{M D}=\frac{1}{8 \pi} \int|H(r)|^{2} d r
\end{aligned}
$$

When a wave with constant wavelength propagates through a photonic crystal it will modulate by the periodicity in that crystal, so that the wave will have different filed distributions around the localized regions. Fundamentally there are two polarizations called transverse electric (TE) mode where the electric field is confined to $\mathrm{x}-\mathrm{y}$ plane $\left(E_{x}, E_{y}, H_{z}\right)$ and transverse magnetic (TM) mode where the magnetic field is confined to $\mathrm{x}-\mathrm{y}$ plane $\left(H_{x}, H_{y}, E_{z}\right)$. These are orthogonal to each other. The transverse $\boldsymbol{D}$ field is given by: 


$$
D_{k}^{w}(\boldsymbol{r})=\frac{1}{|\boldsymbol{k}|} \sum_{\boldsymbol{G}}|\boldsymbol{k}+\boldsymbol{G}| h_{1}(\boldsymbol{G}) \cdot e^{i(\boldsymbol{k}+\boldsymbol{G}) \cdot \boldsymbol{r}} \hat{z}
$$

and the transverse $H$ field is given by:

$$
H_{k}^{w}(\boldsymbol{r})=\sum_{\boldsymbol{G}} h_{1}(\boldsymbol{G}) \hat{z} \cdot e^{i(\boldsymbol{k}+\boldsymbol{G}) \cdot \boldsymbol{r}}
$$

\section{ENERGY BANDS AND DENSITYOF STATES}

The body centered cubic (BCC) crystal structure and irreducible Brillouin zone is presented in figure 1. The Lattice vectors are:

$$
\boldsymbol{a}_{1}=\frac{a}{2}(1,1,-1), \boldsymbol{a}_{2}=\frac{a}{2}(-1,1,1), \boldsymbol{a}_{3}=\frac{a}{2}(1,-1,1) .
$$

where $a$ is the lattice constant. The symmetric points of the irreducible Brillouin zone are:

$$
\Gamma=(0,0,0), \quad \mathrm{N}=\left(0, \frac{1}{4}, 0\right), \quad \mathrm{H}=\left(-\frac{1}{2}, \frac{1}{2}, \frac{1}{2}\right), \quad \mathrm{P}=\left(\frac{1}{4}, \frac{1}{4}, \frac{1}{4}\right)
$$

and these are presented in figure $1(b)$. BCC photonic crystal was formed by replacing the atoms in the primitive cell with dielectric spheres in air as shown in figure $1(c)$ so that the dielectric constants for localized media $\varepsilon_{a}=\varepsilon$ and background material $\varepsilon_{b}=1$ or by drilling air spheres in dielectric mediums $\left(\varepsilon_{a}=1\right.$ and $\left.\varepsilon_{b}=\varepsilon\right)$. The grid points in space were specified in a three dimensional grid and the number of grid points or plane waves used was $(2 n+1)^{3}, n$ been the number of grid points in the direction of each basis lattice vector. Hence the complexity and dimensions of dielectric function was very large compared to two dimensional photonic crystals. Defining the number of plane waves as 343, the reciprocal grid was formed. After specifying the high symmetrical points in the three dimensional geometry, all the coefficients in matrix $M$ of equation 5 were obtained by calculating two unit vectors. Lattice constant was taken as one $(a=1)$. The radius of the dielectric sphere $R$ was changed until the closed packed filling factor of 0.68 was obtained $(R=a \sqrt{3} / 4=0.43 a)$. All the eigenvalues for the matrix were calculated for each $\boldsymbol{k}$ vector in the first irreducible Brillouin zone. There were no complete band gaps for closely packed body centered cubic lattice formed from $\mathrm{SiO}_{2}, \mathrm{GaP}, \mathrm{Si}$, InP, GaAs, InAs, Ge and $\mathrm{BaSrTiO}_{3}$ dielectric spheres drilled in air or for spherical air holes in these dielectric materials. Therefore the filling factor was altered in the range $0.008-0.666$ by changing the radius of the dielectric sphere $(0.1-0.43) a$ untill it was possible to get a complete band gap. For air holes drilled in all the dielectric materials as no complete band gap was found, these are not presented. $\mathrm{SiO}_{2}(\varepsilon=3.9)$, dielectric spheres in air does not give any complete or partial band gap even with the change of the filling factor from $0.008-0.680$ which in turn

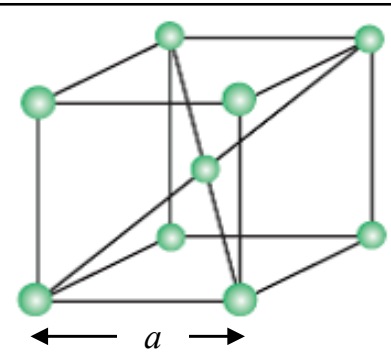

1(a): BCC real space lattice

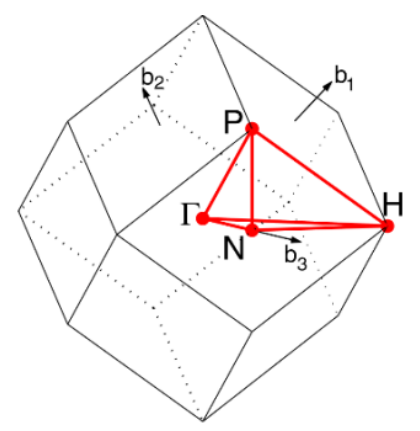

1(b): BCC irreducible Brillouin zone

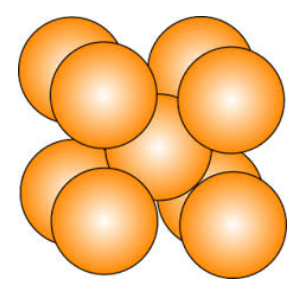

1(c): BCC lattice formed by replacing atoms with dielectric spheres 
changes the radius of the sphere from $(0.10-0.43) a . \mathrm{BaSrTiO}_{3}(\varepsilon=200)$ spheres in air also does not give a complete band gap for any of these filling factors, although there are partial band gaps for different filling factors. Hence these are also not presented.

The calculated energy bands and density of states for optimum band gaps obtained by changing the radius of the spheres in symmetric directions $\Gamma, N, P, H$ of the irreducible Brillouin zone of the body centered cubic (figure 1(b)) formed from dielectrics $\mathrm{GaP}, \mathrm{Si}, \mathrm{InP}, \mathrm{GaAs}$, InAs and $\mathrm{Ge}$ spheres drilled in air are shown in figures 2-7 for normalized frequency $\omega a / 2 \pi c=a / \lambda$. The yellow region indicates a complete gap. Figure 2 shows the energy bands and density of states of BCC $\operatorname{GaP}(\varepsilon=11.1)$ crystal formed from dielectric spheres of radius $R=0.25$ having a filling factor of 0.0736 . GaP has a complete band gap of $0.021 \omega a / 2 \pi c$ between second and third bands and the gap to midgap frequency ratio $\left(\Delta \omega / \omega_{\text {mid }}\right)$ is $4.83 \%$. There is a partial band gap between the sixth and the seventh band. This gap is the largest band gap given by GaP BCC crystal among all the different filling factors. Since the lattice will be in micrometer scale it is a very small lattice. According to the size of the wavelength that propagates through the lattice, the lattice dimensions change. Energy bands and density of states of BCC crystal formed from $\operatorname{Si}(\varepsilon=11.68)$ spheres of radius $R=0.25$ in air is shown in figure 3. This BCC Si lattice has the same filling factor of 0.0736 as GaP. The optimum band gap was obtained between second and third band and there are partial band gaps between fourth and fifth bands and fifth and sixth band. The gap to midgap frequency ratio $\left(\Delta \omega / \omega_{\text {mid }}\right)$ is $4.84 \%$ for the complete band gap.

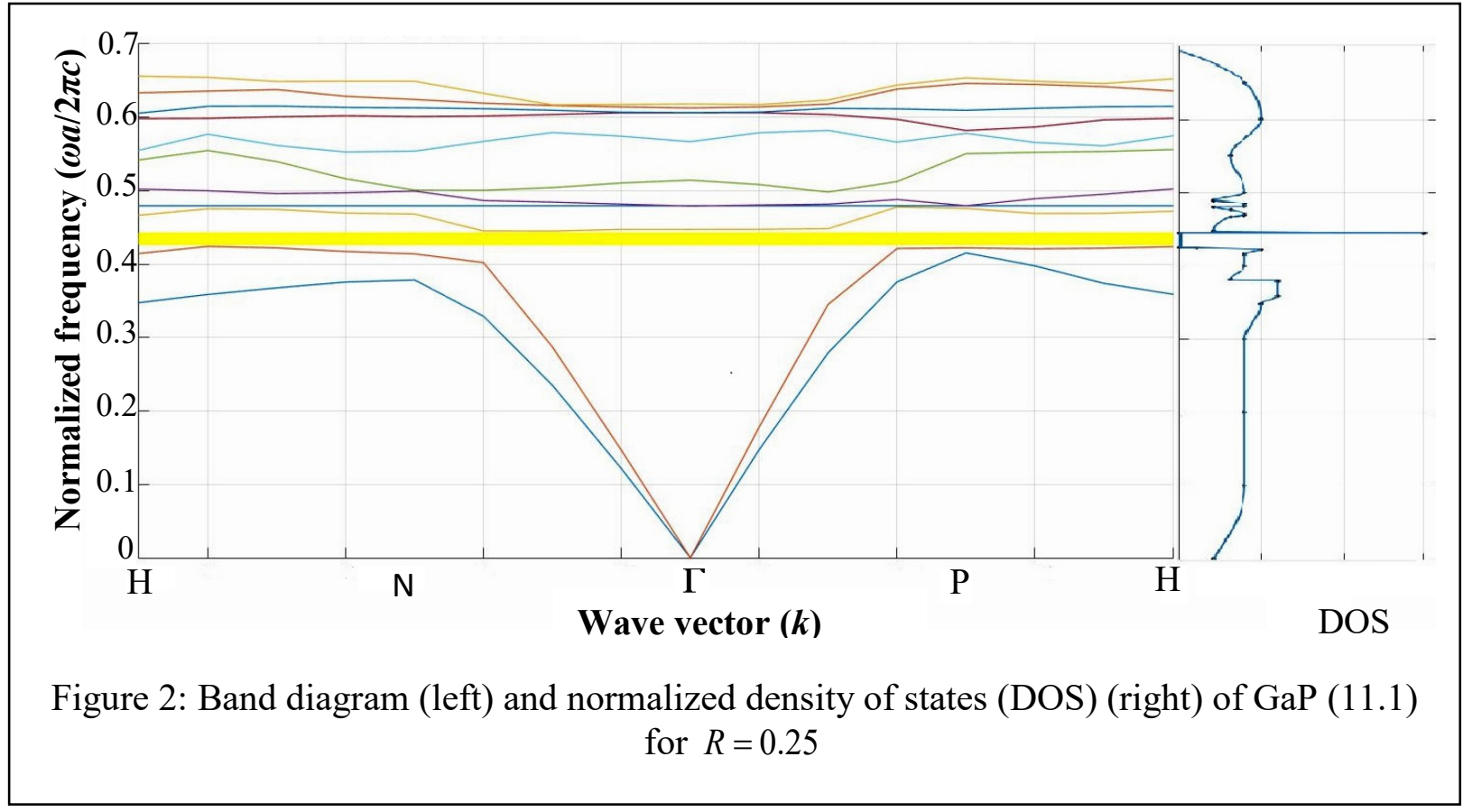




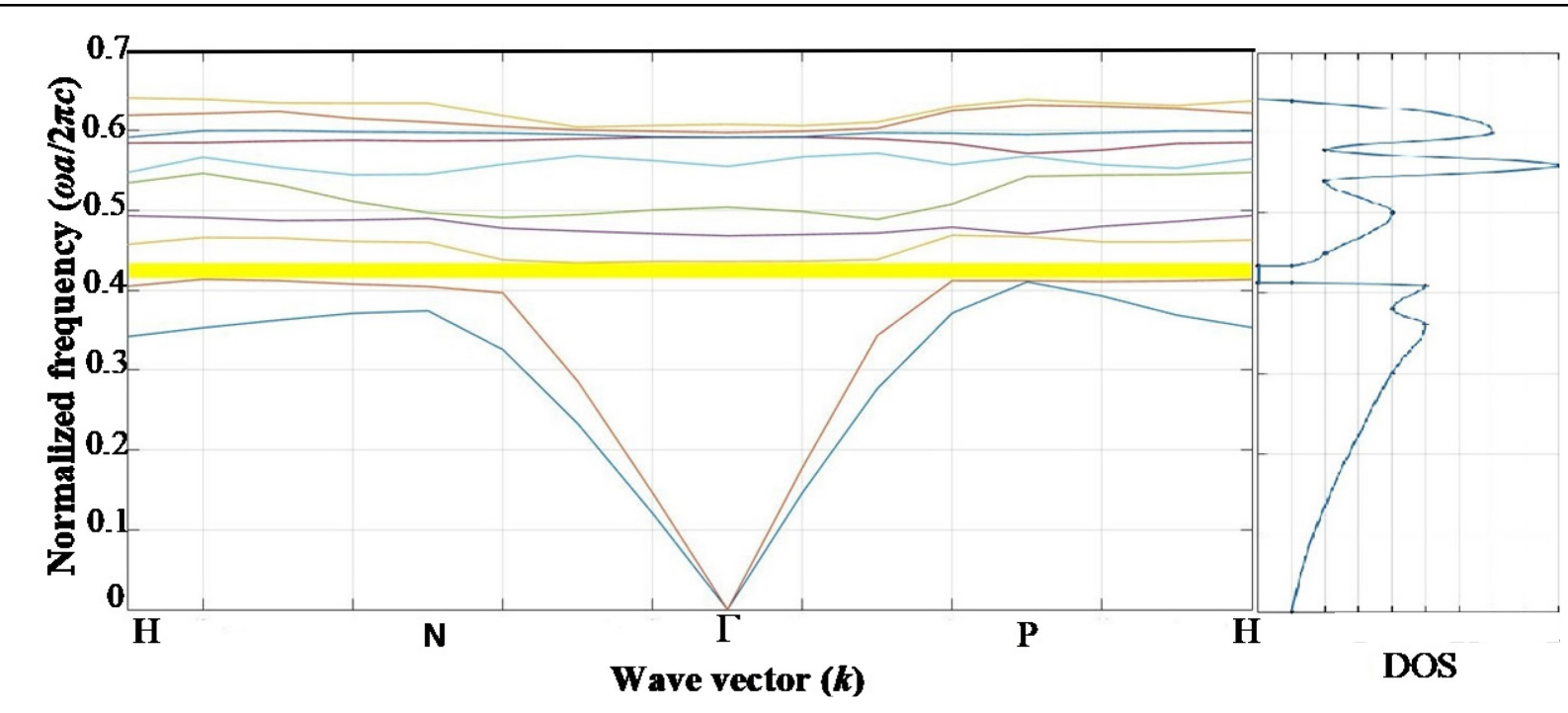

Figure 3: Band diagram (left) and DOS (right) of Si (11.68) for $R=0.25$

Figures 4 and 5 shows the energy bands and density of states of BCC crystal formed from InP $(\varepsilon=12.4)$ and GaAs $(\varepsilon=13.1)$ spheres of radius $R=0.25$ in air respectively. InP lattice has the same filling factor as $\mathrm{GaP}$ and $\mathrm{Si}$. It has a complete band gap of $0.0173 \omega a / 2 \pi c$ between second and third bands and the gap to midgap frequency ratio $\left(\Delta \omega / \omega_{\text {mid }}\right)$ is $4.19 \%$. There are partial band gaps between fourth and fifth bands and fifth and sixth bands. GaAs $(\varepsilon=13.1)$ spheres in air gives a very narrow complete band gap of $0.0136 \omega a / 2 \pi c$ between second and third bands when the filing factor is 0.067 . The gap to midgap frequency ratio $\left(\Delta \omega / \omega_{\text {mid }}\right)$ is $2.75 \%$. There is only one partial band gap between fifth and sixth bands.

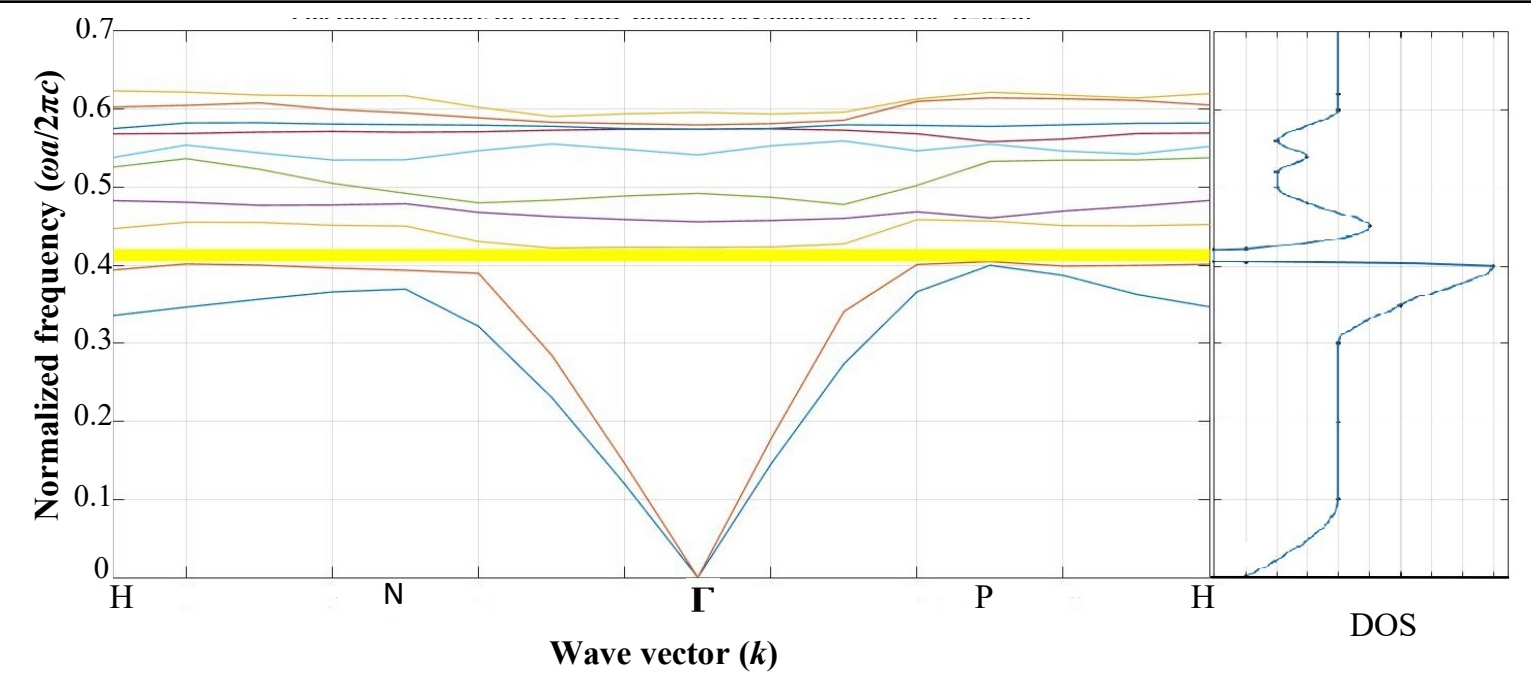

Figure 4: Band diagram (left) and DOS (right) of InP (12.4) for $R=0.25$ 


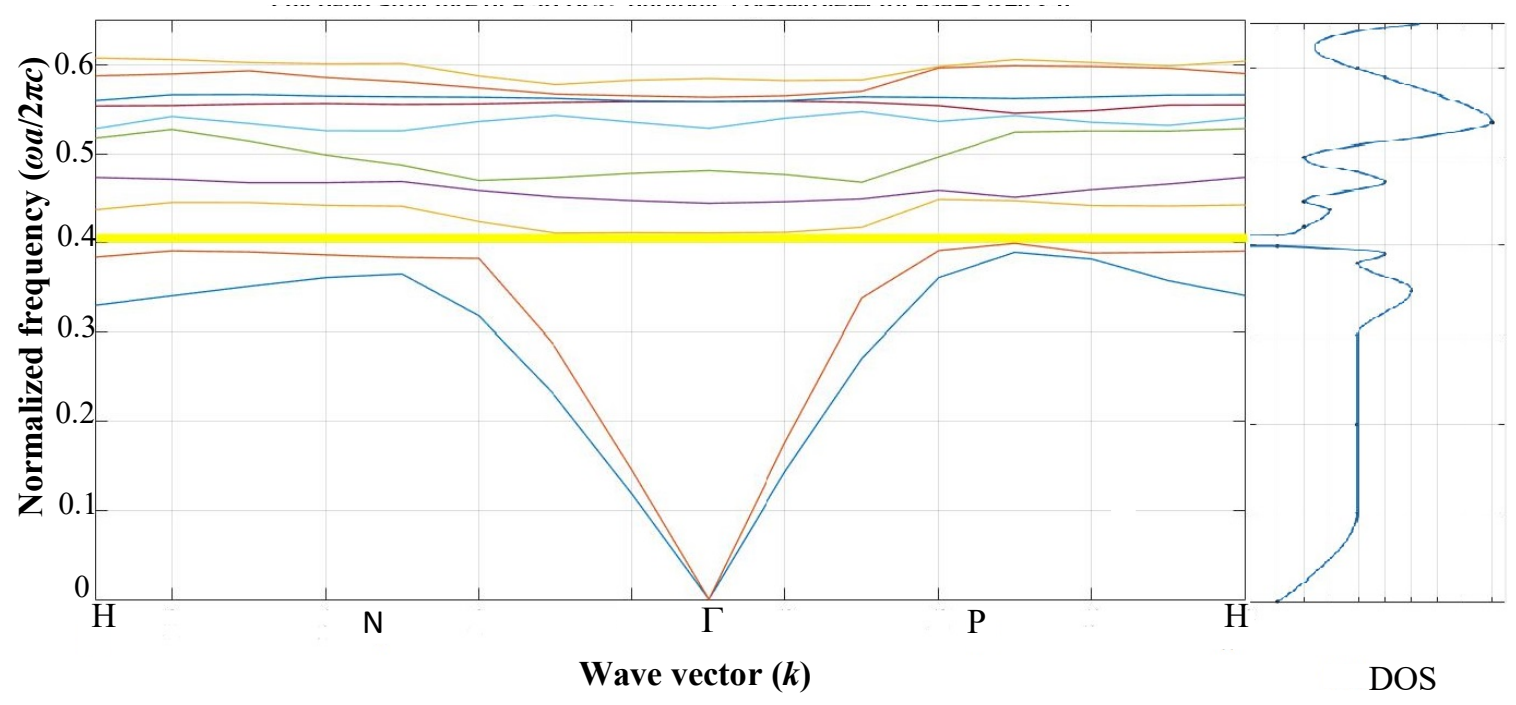

Figure 5: Band diagram (left) and DOS (right) of GaAs (13.1) for $R=0.25$

The energy bands and DOS of BCC structures formed from InAs $(\varepsilon=14.6)$ and Ge $(\varepsilon=16)$, spheres of radius $R=0.2$ drilled in air are shown in figures 6 and 7 respectively. InAs and Ge only gives a complete band gap $0.013 \omega a / 2 \pi c$ between second and third bands when its filling factor is 0.067 and for all other filling factors, a band gap is not obtained. For InAs and Ge the complete band gaps in normalized frequency $\omega a / 2 \pi c$ are 0.013 and 0.0127 respectively and The gap to midgap frequency ratio $\left(\Delta \omega / \omega_{\text {mid }}\right)$ are $2.77 \%$ and $2.83 \%$ respectively. There are partial band gaps between fourth and fifth bands and fifth and sixth bands for both InAs and Ge.

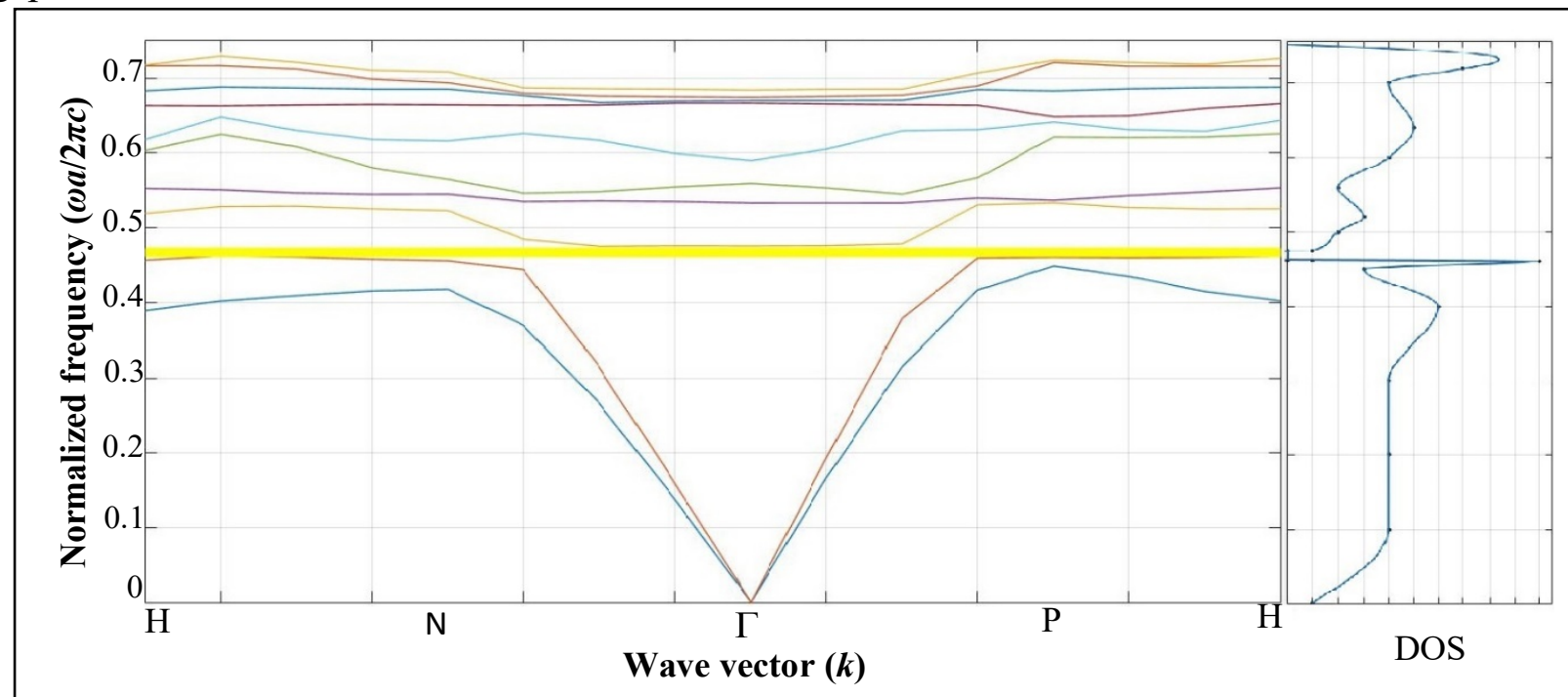

Figure 6: Band diagram (left) and density of states (DOS) (right) of InAs (14.6) for $R=0.2$ 


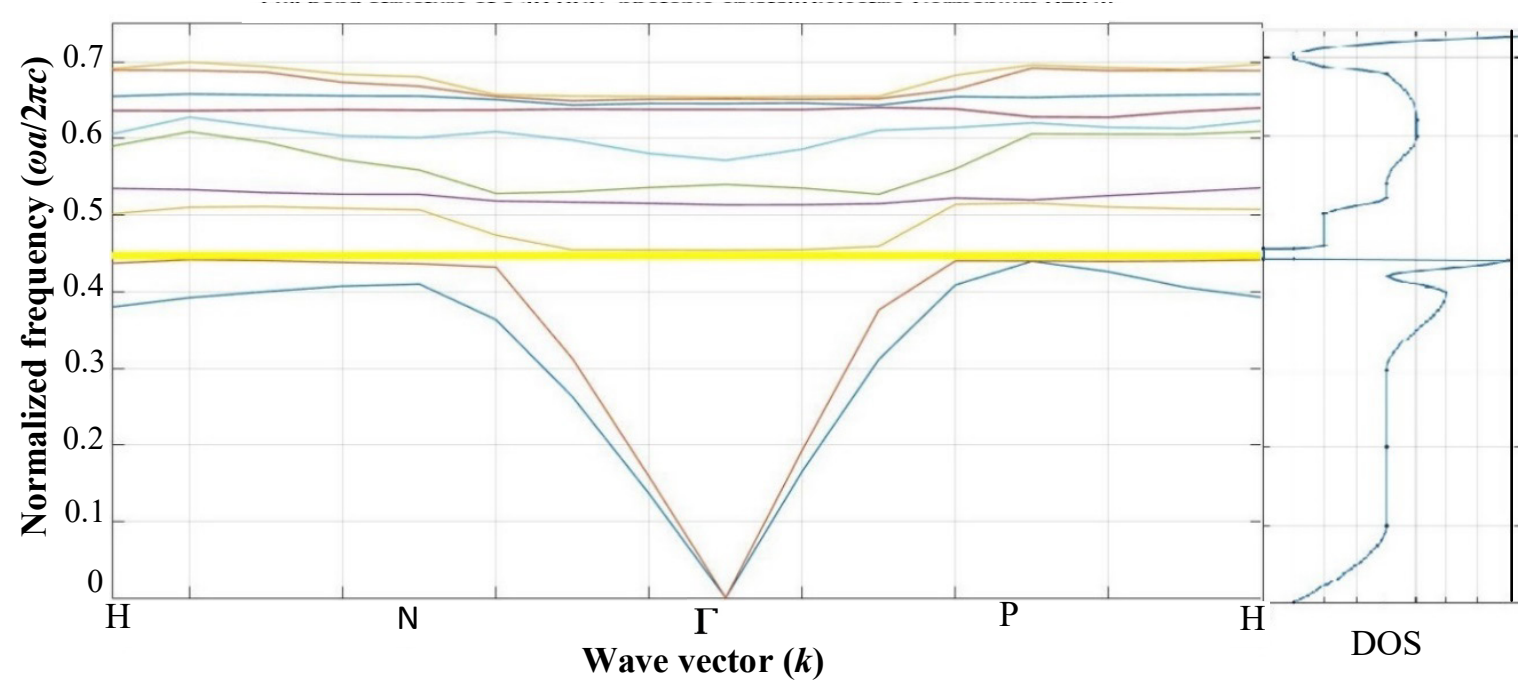

Figure 7: Band diagram (left) and density of states (DOS) (right) of Ge (16) for $R=0.2$

From these energy band diagrams, the gap widths and the midgap widths were obtained for each BCC material. For the telecommunication wave length $\lambda=1.55 \mu \mathrm{m}$, the lattice constants were obtained from the midgap position $a / \lambda$. The radius of spheres for each type of BCC material, filling factors and lattice constants with the width to midgap ratios for $\mathrm{GaP}, \mathrm{Si}, \mathrm{InP}, \mathrm{GaAs}$, InAs and $\mathrm{Ge}$ for telecommunication wavelength are presented in table 1.

Table 1: Lattice parameters, dielectric constants and band gaps for telecommunication wavelength $1.55 \mu \mathrm{m}$

\begin{tabular}{|c|c|c|c|c|c|c|}
\hline Material & $\begin{array}{c}\text { Dielectric } \\
\text { constant }\end{array}$ & $\begin{array}{c}\text { Lattice } \\
\text { constant } \\
\mu \mathrm{m}\end{array}$ & $\begin{array}{c}\text { Radius of } \\
\text { the sphere } \\
\mu \mathrm{m}\end{array}$ & $\begin{array}{c}\text { Filling } \\
\text { factor }\end{array}$ & $\begin{array}{c}\text { Band gap } \\
\omega a / 2 \pi c\end{array}$ & $\begin{array}{c}\text { Gap width to } \\
\text { midgap } \\
\text { frequency ratio }\end{array}$ \\
\hline $\mathrm{GaP}$ & 11.1 & 0.6738 & 0.168 & 0.0736 & 0.0212 & $4.83 \%$ \\
\hline $\mathrm{Si}$ & 11.7 & 0.6571 & 0.164 & 0.0736 & 0.0205 & $4.84 \%$ \\
\hline $\mathrm{GaAs}$ & 13.1 & 0.7654 & 0.191 & 0.0670 & 0.0136 & $2.75 \%$ \\
\hline $\mathrm{InAs}$ & 14.6 & 0.7263 & 0.145 & 0.0670 & 0.0133 & $2.77 \%$ \\
\hline $\mathrm{InP}$ & 12.4 & 0.6407 & 0.164 & 0.0736 & 0.0173 & $4.19 \%$ \\
\hline $\mathrm{Ge}$ & 16.0 & 0.6948 & 0.139 & 0.0670 & 0.0127 & $2.83 \%$ \\
\hline
\end{tabular}

\section{GAP WIDTH WITH DIELCTRIC CONTRAST AND FILLING FACTOR}

The existence and size of a photonic band gap in a photonic crystal is dictated by the symmetry of the crystal and the magnitude of the difference in refractive indices between the constituent materials in the system. The gap width depends on the filling factor and therefore the variation of gap width with the filling factor for the materials which gave complete band gaps were calculated and presented in figure 8. GaP, $\mathrm{Si}, \mathrm{Ga} \mathrm{As}$ and $\mathrm{InP}$ gave the maximum band gaps. For all these materials with complete band gaps, the band gap with the filling factor resembles a $\mathrm{F}$ distribution. When the filling factor is $0.131 \mathrm{GaAs}$ gives the largest band gap relative to other four 
materials which have complete band gaps. InAs and Ge materials give band gaps only for the filling factor of 0.067. For the filling factor of 0.0736, compared to all the dielectrics spheres in air, the largest band gap is given by GaP. Also for filling factors greater than $0.131 \mathrm{GaP}$ always gives the largest band gap although for the filling factors less than 0.131, it gives the third largest band gap. $\mathrm{GaP}, \mathrm{Si}$ and InP materials give band gaps between the filling factor of 0.028 and 0.359 while GaAs gives band gap between the filling factor of 0.0283 and 0.226 . GaP, $\mathrm{Si}, \mathrm{InP}, \mathrm{GaAs}$, InAs and Ge gives a complete photonic band gap for a filling factor of 0.067 . Therefore the filling factor 0.067 can be used for modeling most BCC photonic crystals. Suppose GaP crystal was used in microwave wavelength region, $1 \mathrm{~mm}$ to $1 \mathrm{~m}$ then the lattice constant $a$ will vary from $0.4347 \mathrm{~mm}$ to $0.4347 \mathrm{~m}$. Therefore GaP BCC photonic crystal is suitable to use in microwave region or for long distance telecommunications, GPS or radar etc.

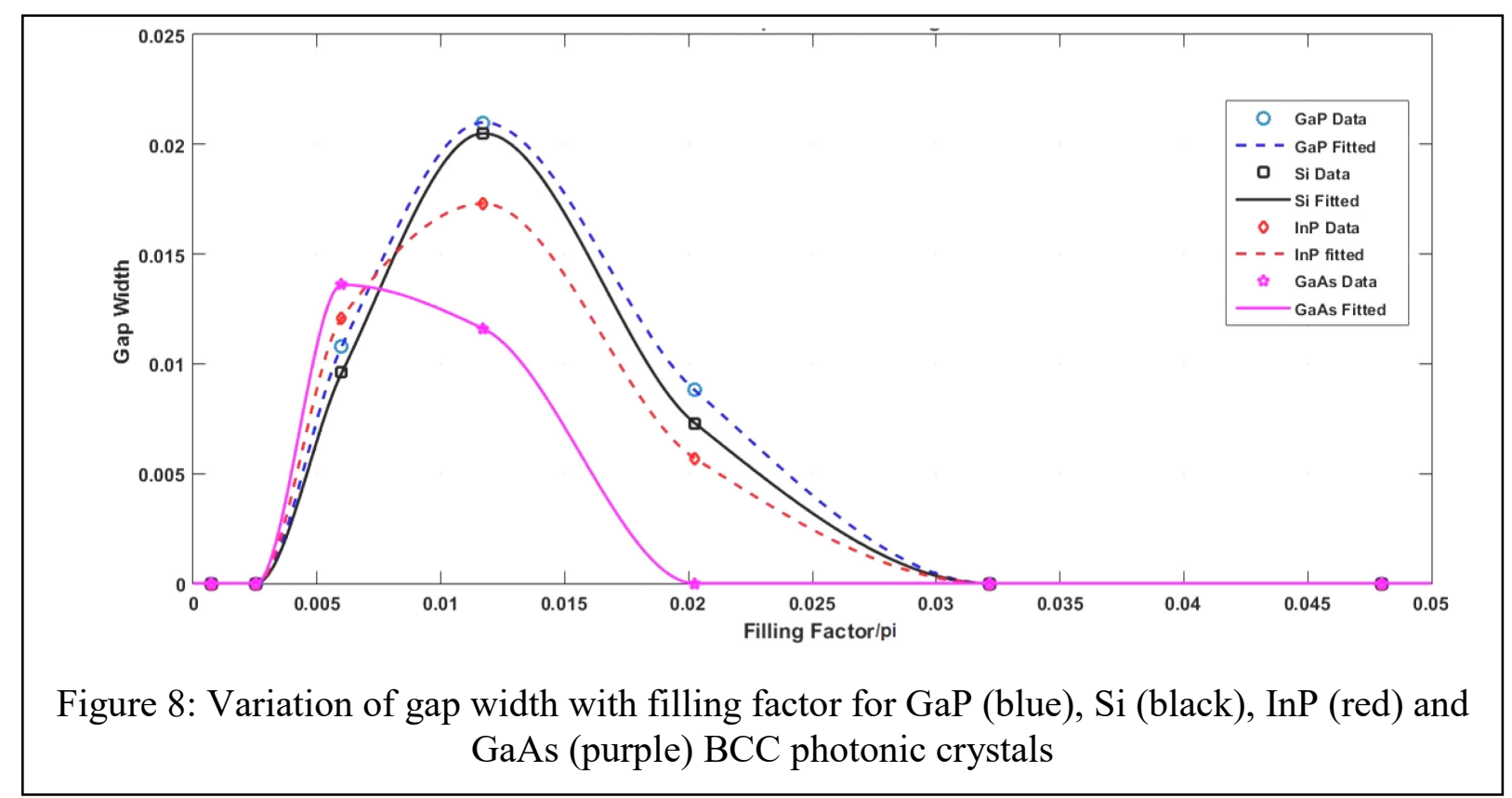

For each crystal structure there is a minimum refractive index contrast, which opens a photonic band gap. In order to increase the size of the gap, the difference in the refractive indices should be maximized. This imposes important restrictions on the photonic crystal structures and constituent materials that may be used in order to generate a photonic band gap. Therefore the variation of the gap width to gap to mid-gap ratio $\Delta \omega / \omega_{\text {mid }}$ with dielectric constant was calculated and presented in figure 9 for BCC photonic crystals formed from dielectric spheres with radius $0.2 a$ (blue), $0.25 a$ (black) and $0.3 a$ (red) in air. It can be seen that $\Delta \omega / \omega_{\text {mid }}$ is decreasing with the dielectric constant for filling factors $0.131(0.25 a)$ and $0.226(0.3 a)$. These filling factors 0.131 and 0.226 give smallest gap-to-mid-gap ratio for GaAs and InP respectively. 


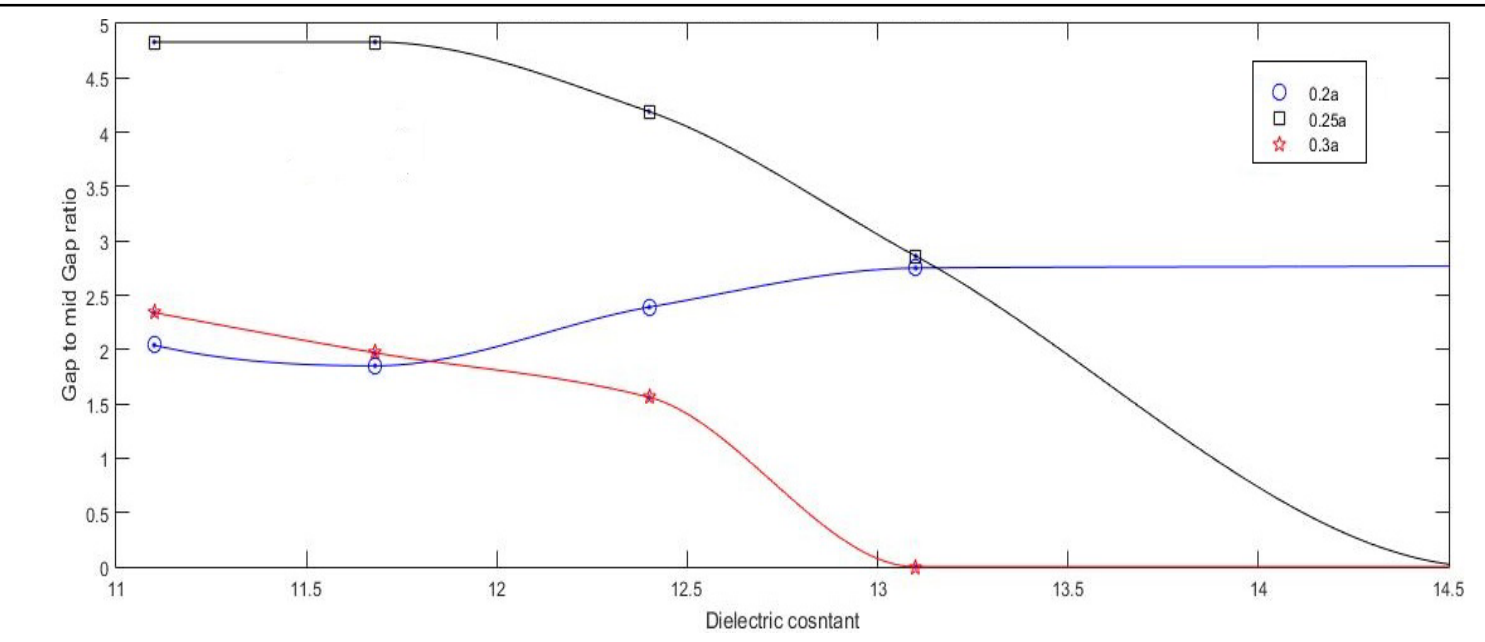

Figure 9: Variation of gap width to midgap ratio with dielectric constant for spheres with radius $0.2 a$ (blue), $0.25 a$ (black) and $0.3 a$ (red)

For the filling factor of 0.067 that is spheres with radius $0.2 a$, the minimum band gap is given by $\mathrm{Si}$, while all the other InP, GaAs, InAs and Ge band gaps increases with dielectric constant. For other filling factors, the band gap decreases with the increase in dielectric constants. The largest gap to midgap ratio is given by GaP. Optimum band gap materials and filling factors for BCC structure for wavelength rage $1550 \mathrm{~nm}-1 \mathrm{~mm}$ is given in table 2 . This BCC structure can be used in radio wave frequency for wavelength range $1 \mathrm{~mm}-100,000 \mathrm{~km}$. The ranges of the lattice constants for different radio wavelength bands of extremely high frequency, EHF $(1 \mathrm{~mm}-1 \mathrm{~cm})$, super high frequency, SHF $(1 \mathrm{~cm}-10 \mathrm{~cm})$, ultra high frequency, UHF $(10 \mathrm{~cm}-1 \mathrm{~m})$ and very high frequency, VHF $(1 \mathrm{~m}-10 \mathrm{~m})$ for GaAs with a filling factor of 0.067 , GaP with a filling factor 0.131 and 0.226 with optimum gaps $0.0136,0.021$ and 0.0088 in normalized frequency respectively are given in table 3 .

Table 2: Optimum band gap materials and filling factors for BCC structure for wavelength range $1550 \mathrm{~nm}-1 \mathrm{~mm}$

\begin{tabular}{|c|c|c|c|}
\hline $\begin{array}{c}\text { Optimum gap in } \\
\omega a / 2 \pi c\end{array}$ & Filling factor & $\begin{array}{c}\text { Range of the lattice } \\
\text { constant }\end{array}$ & Dielectric material \\
\hline 0.0136 & 0.067 & $0.77 \mu \mathrm{m}-0.49 \mathrm{~mm}$ & $\mathrm{GaAs}$ \\
\hline 0.021 & 0.131 & $0.67 \mu \mathrm{m}-0.43 \mathrm{~mm}$ & $\mathrm{GaP}$ \\
\hline 0.0088 & 0.226 & $0.58 \mu \mathrm{m}-0.38 \mathrm{~mm}$ & $\mathrm{GaP}$ \\
\hline
\end{tabular}


Table 3: Optimum band gap materials and filling factors for BCC structure for EHF, SHF, UHF and VHF ranges

\begin{tabular}{|c|c|c|c|}
\hline Dielectric material & GaAs & GaP & GaP \\
\hline $\begin{array}{c}\text { Optimum gap in } \\
\omega a / 2 \pi c\end{array}$ & 0.0136 & 0.021 & 0.0088 \\
\hline Filling factor & 0.067 & 0.131 & 0.226 \\
\hline $\begin{array}{c}\text { Lattice constant for } \\
\text { EHF }(1 \mathrm{~mm}-1 \mathrm{~cm})\end{array}$ & $0.49 \mathrm{~mm}-0.49 \mathrm{~cm}$ & $0.43 \mathrm{~mm}-0.43 \mathrm{~cm}$ & $0.38 \mathrm{~mm}-0.38 \mathrm{~cm}$ \\
\hline $\begin{array}{c}\text { Lattice constant for } \\
\text { SHF }(1 \mathrm{~cm}-10 \mathrm{~cm})\end{array}$ & $0.49 \mathrm{~cm}-4.9 \mathrm{~cm}$ & $0.43 \mathrm{~cm}-4.3 \mathrm{~cm}$ & $0.38 \mathrm{~cm}-3.8 \mathrm{~cm}$ \\
\hline $\begin{array}{c}\text { Lattice constant for } \\
\text { UHF }(10 \mathrm{~cm}-1 \mathrm{~m})\end{array}$ & $4.9 \mathrm{~cm}-0.49 \mathrm{~m}$ & $4.3 \mathrm{~cm}-0.43 \mathrm{~m}$ & $3.8 \mathrm{~cm}-0.38 \mathrm{~m}$ \\
\hline $\begin{array}{c}\text { Lattice constant for } \\
\text { VHF }(1 \mathrm{~m}-10 \mathrm{~m})\end{array}$ & $0.49 \mathrm{~m}-4.9 \mathrm{~m}$ & $0.43 \mathrm{~m}-4.3 \mathrm{~m}$ & $0.38 \mathrm{~m}-3.8 \mathrm{~m}$ \\
\hline
\end{tabular}

\section{WAVE PROPERGATION THROUGH GaP BCC PHOTONIC CRYSTAL}

The spreading of the dielectric constant over $\mathrm{x}-\mathrm{Z}$ plane for a BCC crystal formed from $\mathrm{GaP}$ spheres of radius $R=0.25$ in air with a filling factor of 0.0736 is shown in figure 10 . When an electromagnetic wave of wave of wavelength $1.55 \mu \mathrm{m}$ that is a telecommunication wave was propagating through this photonic crystal lattice, the electric field components $E_{x}, E_{y}, E_{z}$ and magnetic field components $H_{x}, H_{y}, H_{z}$ were obtained in a $3 \times 3 \times 3$ photonic cell for transverse electric (TE) mode and transverse magnetic (TM) mode. The amplitudes of these electric and magnetic components are shown in figures 11 and 12 respectively. The amplitude of the electric field around the dielectric spheres is greater than the electric field inside the dielectric spheres. This means electric field will not propagate through the dielectric spheres. When it comes to magnetic field $(H)$, the amplitude inside the spheres is higher than the outside. Therefore magnetic field propagates through the dielectric and will avoid propagation through air.

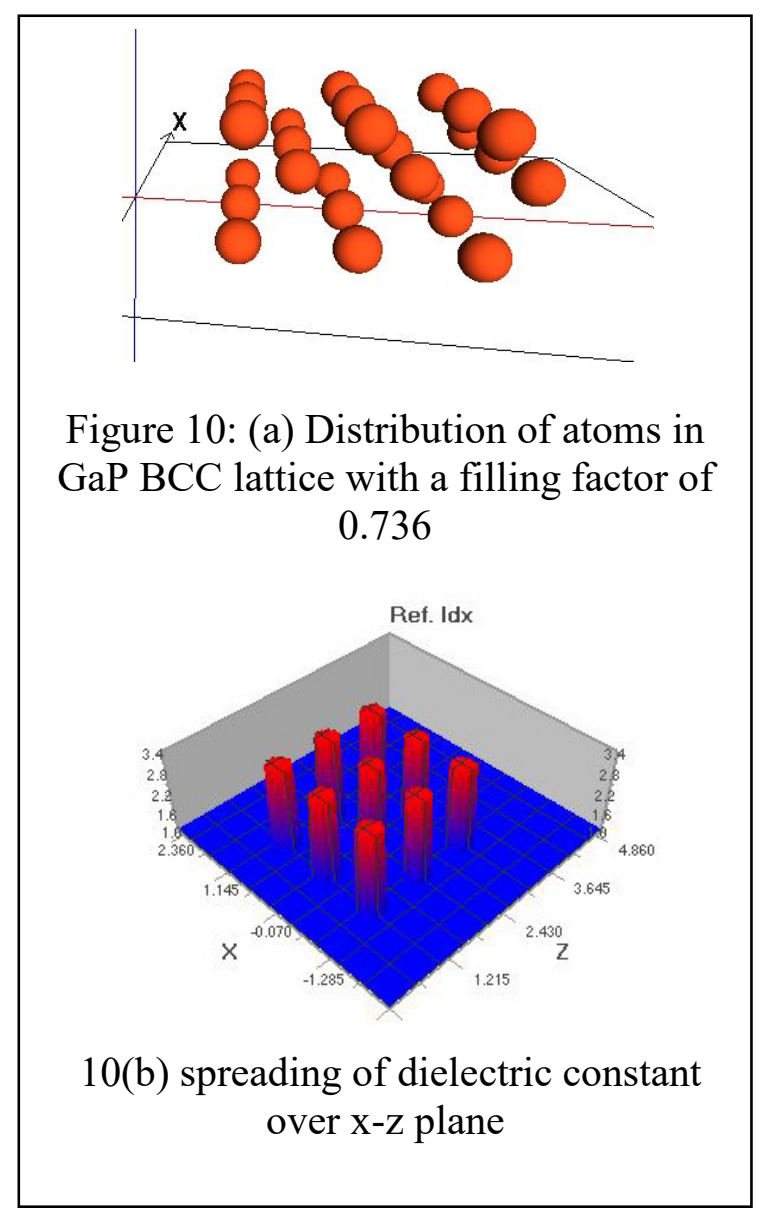




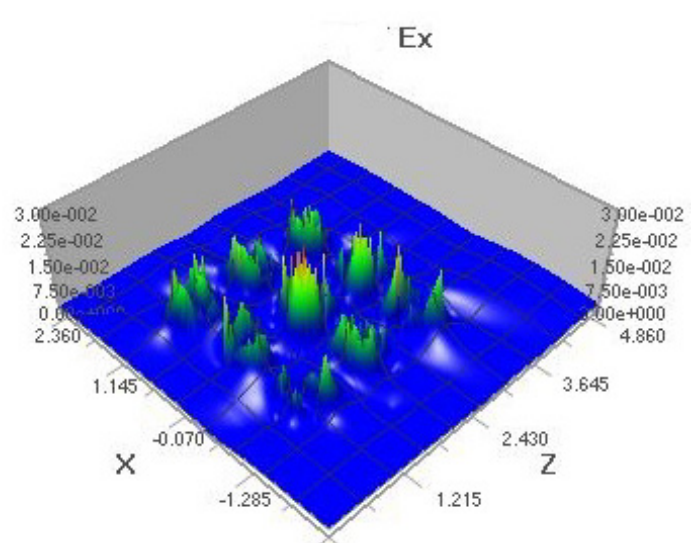

(a)

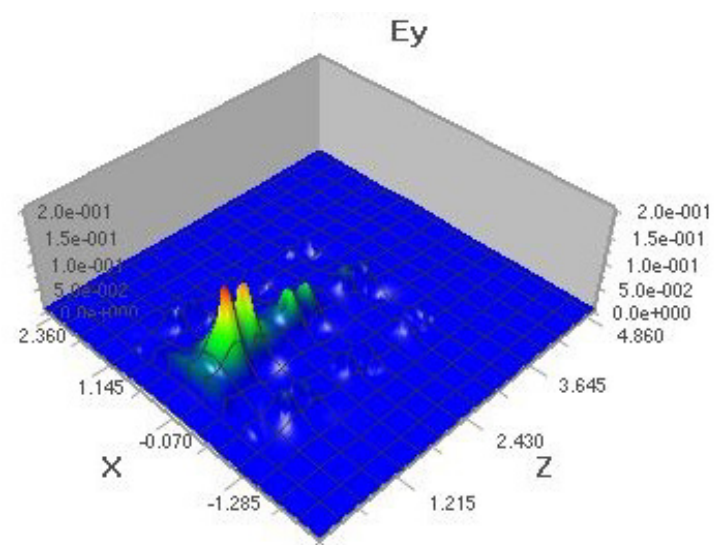

(b)

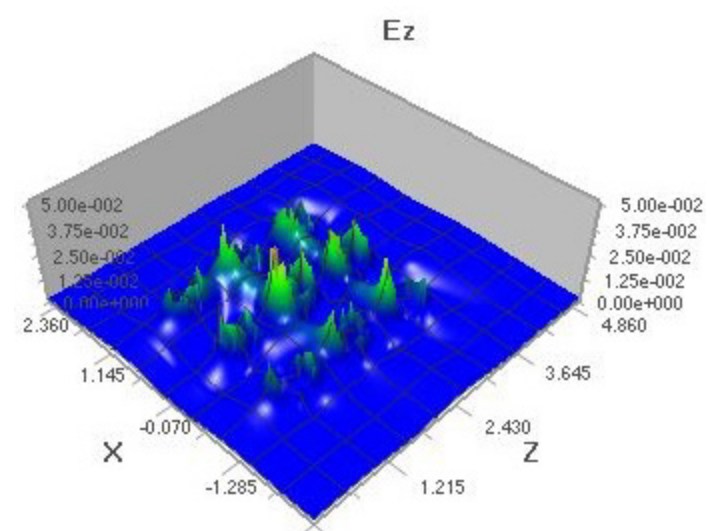

(c)

Figure 11: Propagation of electric field through GaP BCC lattice with filling factor of 0.0736 . The amplitude variations of (a) $E_{x}(b) E_{y}(c) E_{z}$

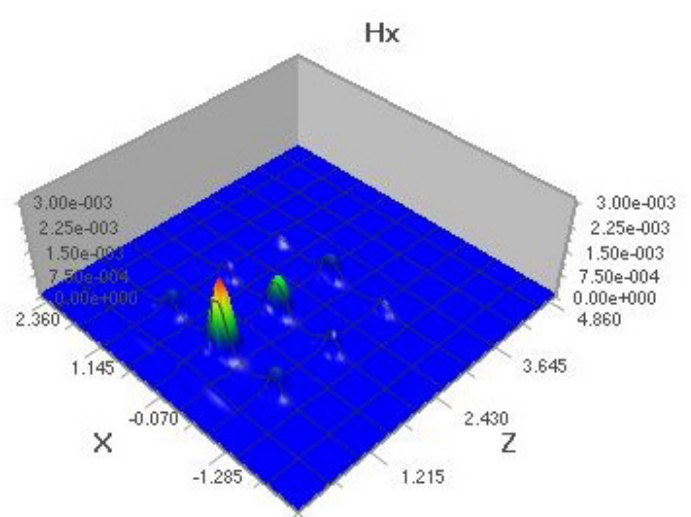

(a)

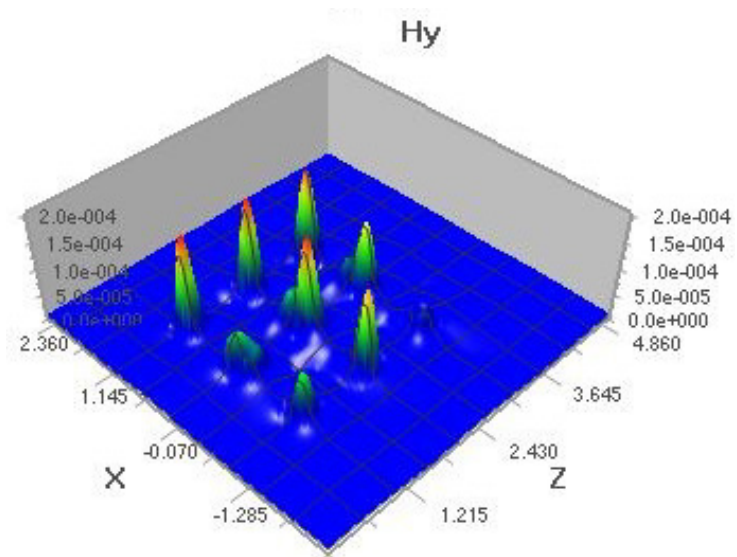

(b)

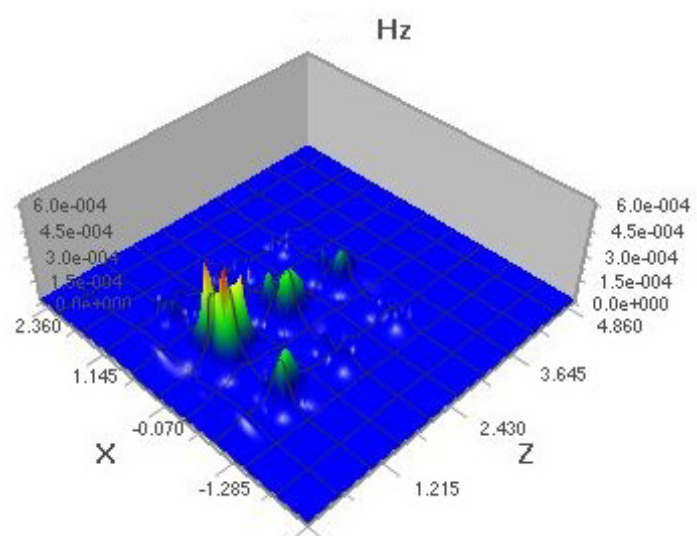

(c)

Figure 12: Propagation of magnetic field through $\mathrm{GaP}$ BCC lattice with filling factor of 0.0736 . The amplitude variations of (a) $H_{x}(b) H_{y}(c) H_{z}$

\section{CONCLUSIONS}

There were no complete band gaps for closely packed body centered cubic lattice formed from $\mathrm{SiO}_{2}, \mathrm{GaP}, \mathrm{Si}, \mathrm{InP}, \mathrm{GaAs}$, InAs, Ge and $\mathrm{BaSrTiO}_{3}$ dielectric spheres drilled in air or for spherical air holes in these dielectric materials. Therefore the filling factor was altered by changing the radius of the dielectric sphere and it was possible to get complete band gaps for GaP, Si, InP, GaAs, InAs, and $\mathrm{Ge}$ for a filling factor of 0.067 , but not for $\mathrm{SiO}_{2}$ and $\mathrm{BaSrTiO}_{3}$. The most significant band gap 
was given by $\mathrm{Si}$ closely followed by $\mathrm{GaP}$ for a filling factor of 0.0736 . All the crystals can be designed in nanometer scale if they are utilized in telecommunication wavelength, but if they are utilized in microwave wavelength they can be designed in the rage of millimeter scale to meter scale. For the wavelength rage $1550 \mathrm{~nm}-1 \mathrm{~mm}$, the optimum band gap of 0.021 normalized frequency was obtained for $\mathrm{GaP}$ for the filling factor of 0.131 with radius of the spheres ranging from $0.67 \mu \mathrm{m}-0.43 \mathrm{~mm}$ and a band gap of 0.0136 normalized frequency for GaAs for a filling factor of 0.067 with radius of the spheres ranging from $0.77 \mu \mathrm{m}-0.49 \mathrm{~mm}$. Therefore $\mathrm{GaP}$ and $\mathrm{GaAs}$ are suitable to use in microwave region or for long distance telecommunications, GPS and radar. Mode field distributions of TE and TM mode obtained for BCC lattice formed from GaP spheres of radius 0.25 in air with filling factor of 0.0736 showed that for telecommunication wavelength, the electric field will not propagate through the dielectric spheres while the magnetic field propagates through the dielectric spheres avoiding the propagation through air.

\section{References:}

[1] V. P. Bykov, Spontaneous emission from a medium with a band spectrum, Quantum Electronics, vol. 4, no. 7, pp. 861-871, 1975.

[2] K. Ohtaka, Energy band of photons and low-energy photon diffraction, Phys. Rev. B vol. 19, no. 10, pp. 5057-5067, 1979.

[3] E .Yablonovich, Inhibited spontaneous emission in solid-state physics and electronics, Phys. Rev. Lett., vol. 58, pp. 2059-2062, 1987.

[4] S. John, Strong localization of photons in certain disordered dielectric superlattices, Phys. Rev. Lett., vol. 58, pp. 2486-2489, 1987.

[5] E. Yablonovitch, T. J. Gmitter, K. M. Leung, Photonic band structure: the face-centered-cubic case employing nonspherical atoms, Phys. Rev. Lett., vol. 67, no. 17, pp. 2295-2298, 1991.

[6] J. D Joannopoulos, S. G. Johnson, J. N. Winn and R. D. Meade, Photonic Crystals: Molding the Flow of Light. 2nd ed. Princeton: Princeton University press, 2008.

[7] S. Guo, S. Albin, Simple plane wave implementation for photonic crystal calculations, Optics Express, vol.11, no. 2, p. 167, 2003.

[8] A. J. Danner, "An introduction to the plane wave expansion method for calculating photonic crystal band diagrams", University of Illinois at Urbana-Champaign, Urbana, 2011. Available at: https://www.ece.nus.edu.sg/stfpage/eleadj/planewave.htm. 